\title{
Efecto de una intervención educativa a escolares con sobrepeso y obesidad
}

\section{Effect of an educational intervention on fifth-year primary school children with overweight and obesity}

\author{
Rosa M. Baltazar Téllez, ${ }^{a}$, Anely Martínez Francisco ${ }^{b}$, Esther Ramírez Moreno ${ }^{c}$, \\ José Arias Rico ${ }^{d}$, Rosa M. Guevara Cabrera ${ }^{e}$, Rosario Barrera Gálvez ${ }^{f}$, \\ C. Efrén Hernández Hernández ${ }^{g}$, Alma B. López Escudero ${ }^{h}$
}

\begin{abstract}
:
In Mexico and Hidalgo occupy the first place worldwide in childhood obesity, the World Health Organization, 2017 states that the promotion of healthy diets and regular physical activity are essential factors in the fight against the epidemic of childhood obesity. Methodology.- a quantitative, correlational, quasi-experimental, (pre-post), longitudinal study was carried out, the correct identification of the dish of good eating was evaluated, as well as physical activity, knowledge about the complications of overweight, obesity and obesity. Change of BMI after intervention. Results.- In physical activity, soccer is the sport most played by schoolchildren and there is an increase in post intervention of $5.91 \%$. In the pre-intervention, $82.35 \%$ did physical activity and in the post-intervention it increased to $88.24 \%$. According to the pre-intervention results, it was obtained that only $17.65 \%$ of school children correctly identify the plate of good eating, after intervention it was obtained that $88.24 \%$ of the schoolchildren learned to correctly identify them, the BMI of the 35 schoolchildren was that the $17.10 \%$ presented low weight, $34.30 \%$ healthy weight, $31.40 \%$ were overweight and $17.20 \%$ had obesity. Post Intervention to the 17 schoolchildren with overweight and obesity was obtained that $64.70 \%$ presented healthy weight, $23.50 \%$ overweight and $11.80 \%$ presented. Conclusions.- There is a statistically significant effect in the intervention carried out on schoolchildren with overweight and obesity in the reduction of BMI.
\end{abstract}

Keywords:

Scholars, Obesity, overweight - educational intervention

Resumen:

En México e Hidalgo ocupan el primer lugar mundial en obesidad infantil, la Organización Mundial de la Salud, 2017 refiere que el fomento de las dietas saludables y la actividad física regular son factores esenciales en la lucha contra la epidemia de obesidad infantil. Metodología.- se realizó un estudio cuantitativo, correlacional, cuasi experimental, (pre-post), longitudinal, se evaluó la identificación correcta del plato del buen comer, la realización de activad física, el conocimiento sobre las complicaciones de sobrepeso, obesidad y el cambio de IMC pos intervención. Resultados.- En la actividad física el fútbol es el deporte que más realizan los escolares y se presenta un aumento en la pos intervención del 5.91\%. En la pre intervención el $82.35 \%$ sí realiza actividad física y en la pos intervención aumento al $88.24 \%$. De acuerdo a los resultados pre intervención se obtuvo que solo el $17.65 \%$ de los escolares identifica correctamente el plato del buen comer, pos intervención se obtuvo que el $88.24 \%$ de los escolares aprendió a identificarlos correctamente, el IMC de los 35 escolares fue que el $17.10 \%$ presenta bajo peso, el $34.30 \%$ peso saludable, el $31.40 \%$ presentaban sobrepeso y el $17.20 \%$ presentaba obesidad. Post intervención a los 17 escolares con sobrepeso y obesidad se obtuvo que el $64.70 \%$ presenta peso saludable, el $23.50 \%$ sobrepeso y el $11.80 \%$ presenta. Conclusiones.- Existe un efecto estadísticamente significativo en la intervención realizada a los escolares con sobrepeso y obesidad en la disminución del IMC.

\section{Palabras Clave:}

Escolares, Obesidad, sobrepeso - intervención educativ

\footnotetext{
${ }^{a}$ Autora de correspondencia, Universidad Autónoma del Estado de Hidalgo, Instituto de Ciencias de la Salud, ORCID: 0000-0002-21687564, Email: rbaltazartellez@yahoo.com.mx

bUniversidad Autónoma del Estado de Hidalgo, Instituto de Ciencias de la Salud, Email: anely_1989@ hotmail.com ${ }^{c}$ Universidad Autónoma del Estado de Hidalgo, Instituto de Ciencias de la Salud, ORCID: 0000-0002-9928-8600, Email: esther_ramirez@uaeh.edu.mx

${ }^{\mathrm{d}}$ Universidad Autónoma del Estado de Hidalgo, Instituto de Ciencias de la Salud, ORCID: 0000-0003-0219-0410,

Email: josearias.rico@hotmail.com

${ }^{e}$ Universidad Autónoma del Estado de Hidalgo, Instituto de Ciencias de la Salud, ORCID: 0000-0002-1309-2747,

Email: rosi_guevara29@hotmail.com

${ }^{\mathrm{f}}$ Universidad Autónoma del Estado de Hidalgo, Instituto de Ciencias de la Salud, ORCID: 0000-0002-1949-5424,

Email: rosario_barrera@uaeh.edu.mx

${ }^{g}$ Universidad Autónoma del Estado de Hidalgo, Instituto de Ciencias de la Salud, Email: efrenhudson4@gmail.com

h'Universidad Autónoma del Estado de Hidalgo, Instituto de Ciencias de la Salud, Email: belem_0924@ hotmail.com
} 


\section{Marco Teórico}

El país con mayor índice de obesidad es EEUU., seguido por México, Nueva Zelanda y Hungría; en contraparte, los países con índices más bajos de obesidad son Japón y Corea. Se estima que para el año 2030, los índices de obesidad crecerán más, y serán Suiza y Corea las naciones en las que las tasas aumentarán con mayor rapidez. La desigualdad social, la falta de oportunidades para educarse y las diferencias de género, también se manifiestan en el problema de la obesidad. En aproximadamente ocho de los países de los que se tiene información confiable, las mujeres menos educadas son entre dos y tres veces más propensas a desarrollar sobrepeso, que en las naciones en las que las mujeres tienen un mayor nivel educativo. Los niños obesos y con sobrepeso tienden a seguir siendo obesos en la edad adulta y tienen más probabilidades de padecer a edades más tempranas enfermedades no transmisibles como la diabetes y las enfermedades cardiovasculares. (Alleyne, 2016). De acuerdo a la Organización Mundial de la Salud (OMS) el fomento de las dietas saludables y la actividad física regular son factores esenciales en la lucha contra la epidemia de obesidad infantil. Los padres pueden influir en el comportamiento de sus hijos poniendo a su disposición en el hogar bebidas y alimentos saludables y apoyando y alentando la actividad física. ${ }^{2}$

La Niñez Intermedia es una etapa del desarrollo que comprende de los seis a los once años, donde se aprecian distintos cambios; tantos físicos, psicológicos, cognitivos y sociales. La fuerza y las habilidades atléticas mejoran, las enfermedades respiratorias son comunes pero, en general, la salud es mejor que en cualquier otra época de la vida, el egocentrismo disminuye. La calidad de las relaciones familiares sigue ejerciendo una gran influencia sobre el ajuste emocional y social. ${ }^{3}$

El desarrollo cognitivo desde el enfoque Piagetiano (712 años) habla de la etapa de las operaciones concretas, en donde los procesos de razonamiento se vuelven lógicos y pueden aplicarse a problemas concretos o reales. Los niños de hoy son menos activos que en años anteriores. Se debe desarrollar hábitos y destrezas de bienestar físico para toda la vida puede reducir los riesgos de sobrepeso, obesidad, riesgos cardiovasculares mediante el control de la presión arterial y del colesterol. ${ }^{4}$ El sobrepeso y la obesidad es una acumulación anormal o excesiva de grasa que puede ser perjudicial para la salud. Para la identificación de sobrepeso y obesidad se utiliza el índice de masa corporal (IMC) es un indicador simple de la relación entre el peso y la talla. ${ }^{2}$ Ambas enfermedades son consideradas factor de riesgo que puede conllevar a la muerte a nivel mundial. ${ }^{1}$ Se clasifica como sobrepeso cuando el IMC es igual o superior a 25 y la obesidad cuando éste es igual o superior a $30 .^{2}$ En el caso de los niños de 5 a 19 años, el sobrepeso y la obesidad se definen de la siguiente manera: El sobrepeso es el IMC para la edad con más de una desviación típica por encima de la mediana establecida en los patrones de crecimiento infantil. El exceso de peso corporal (sobrepeso y obesidad) se reconoce actualmente como uno de los retos más importantes de la Salud Pública a nivel mundial debido a su magnitud, la rapidez de su incremento y el efecto negativo que ejerce sobre la salud de la población que la padece. El sobrepeso y la obesidad, incrementan significativamente el riesgo de padecer enfermedades crónicas no transmisibles, la mortalidad prematura y el costo social de la salud. ${ }^{5}$ Los niños obesos tienen más probabilidades de desarrollar una serie de problemas de salud en la edad adulta, consecuencias médicas y psicológicas desde temprana edad como las cardiopatías, resistencia a la insulina, trastornos osteomusculares (especialmente artrosis, una enfermedad degenerativa muy discapacitante que afecta las articulaciones), algunos tipos de cáncer (endometrio, mama y colon) o discapacidad (OMS, 2018). ${ }^{6}$

La intervención educativa que proporciona el profesional de enfermería sobre alimentación saludable y actividad física a los escolares y padres de familia de la escuela primaria "Once de Julio", ayudará a lograr mejorar su nivel de salud, al asumir un mayor control sobre sí mismas, adoptando y ejecutando conductas saludables, logrando un beneficio a su persona para mantener un peso normal y gozar de una alimentación saludable. Nola Pender, autora del Modelo de Promoción a la Salud (MPS), expresó que la conducta está motivada por el deseo de alcanzar el bienestar y el potencial humano. Se interesó en la creación de un modelo enfermero que diera respuestas a la forma como las personas adoptan decisiones acerca del cuidado de su propia salud. La promoción de la salud es la estrategia para realizar diversos planteamientos relacionados con la formulación de políticas públicas saludables, intervenciones dirigidas a personas y comunidades, para la propuesta y realización de investigaciones en salud. Otros conceptos centrales del modelo se relacionan con los conocimientos y afectos (sentimientos, emociones, creencias) específicos de la conducta, comprende seis conceptos: de los cuales se retomó el primero que corresponde a los beneficios percibidos por la acción, que son los resultados positivos anticipados que se producirán como expresión de la conducta de la salud. ${ }^{7}$

\section{Objetivo}

Evaluar el efecto de intervención educativa a los escolares de quinto año, con sobrepeso y obesidad en la escuela primaria Once de Julio, aplicado por el profesional de enfermería.

\section{Material y Método}

Estudio descriptivo correlacional, longitudinal, cuasi experimental (pre-post). (35 alumnos). $n=17$. Se les invitó a los padres de familia a asistir a unas pláticas en la escuela primaria "Once de Julio", sobre la importancia del plato del buen comer, actividad física y las complicaciones de sobrepeso y obesidad en sus hijos. 
Los temas a impartirles fueron: Educación para la salud, Definición de sobrepeso y obesidad, prevalencia, índice de masa corporal, Factores de riesgo que condicionan la aparición de sobrepeso y obesidad, Complicaciones del sobrepeso y obesidad, Impacto psicosocial en el niño con sobrepeso y obesidad, Combatir la obesidad infantil en base al conocimiento, Alimentación saludable, Disminuir y evitar el sedentarismo.

\section{Resultados}

El índice de masa corporal (IMC) es un indicador simple de la relación entre el peso y la talla que se utiliza frecuentemente para identificar el sobrepeso y la obesidad en los adultos. Para determinar la obesidad y sobrepeso en el escolar se utilizaron las tablas para percentilar el IMC a cada escolar. Los resultados obtenidos de los 35 escolares fue que el $17.10 \%$ presenta bajo peso, el $34.30 \%$ peso saludable, el $31.40 \%$ presentaban sobrepeso y el $17.20 \%$ presentaba obesidad. Después de la Intervención Educativa a los 17 escolares con sobrepeso y obesidad se obtuvo como resultado que el $64.70 \%$ presenta peso saludable, el $23.50 \%$ sobrepeso y el $11.80 \%$ presenta obesidad.

Lo cual representa que existe una disminución de escolares con sobrepeso y obesidad.

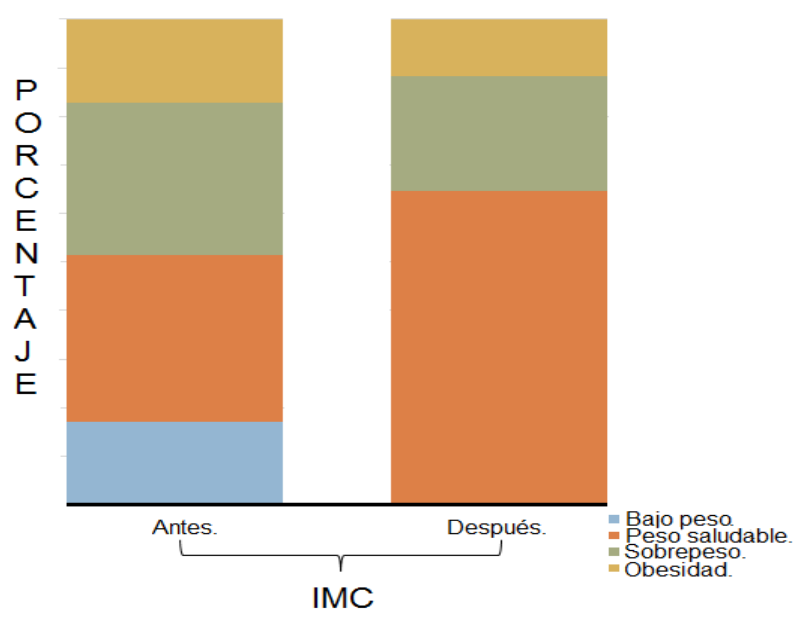

Fuente: IEESO

$n=17$

Solo $22.6 \%$ consumen regularmente verduras, $45.7 \%$ frutas, $60.7 \%$ leguminosas. En cambio se observó un elevado uso de alimentos cuyo gasto cotidiano aumenta los riesgos de obesidad o enfermedades crónicas (grupos de alimentos no recomendables para consumo cotidiano): $81.5 \%$ consumen regularmente bebidas azucaradas no lácteas, $61.9 \%$ botanas, dulces y postres y $53.4 \%$ cereales dulces.

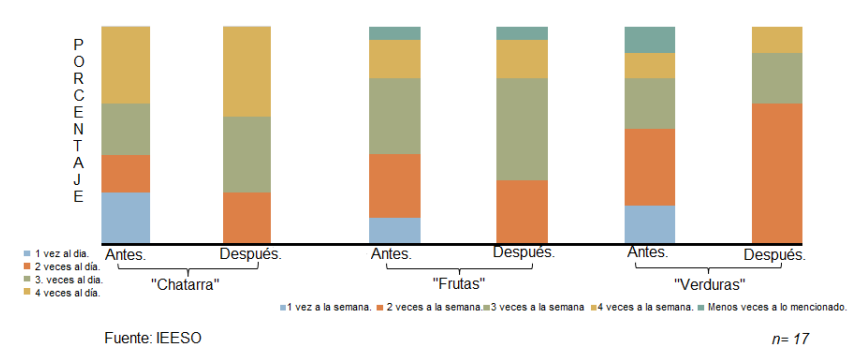

De acuerdo a los resultados pre intervención se obtuvo que solo el $17.65 \%$ de los escolares identifica correctamente el plato del buen comer. Posterior a la Intervención educativa del profesional de Enfermería se obtuvo que el $88.24 \%$ de los escolares aprendió a identificarlos correctamente.

Otro de los factores que contribuye a la prevención del sobrepeso y la obesidad es la actividad física- deportiva, tanto en niños como en adultos. Los hábitos que se adquieren durante la infancia tienden a mantenerse durante el resto de la vida, por eso es importante enseñar a los niños a tener un estilo de vida saludable desde que son pequeños. Los niños y los adolescentes necesitan al menos 60 minutos de actividad física de moderada a intensa la mayoría de los días para el mantenimiento de una buena salud, un buen estado físico y para tener un peso saludable durante el crecimiento. Afortunadamente los resultados que se obtuvieron en la pre intervención fue que el $82.35 \%$ realizan actividad física-deportiva y solo el $17.65 \%$ no realiza actividad física-deportiva. Se observó que en la pos intervención educativa a los escolares existió un aumento, siendo el $88.24 \%$ los que sí realizan actividad física-deportiva y solo el $11.76 \%$ no lo realiza.

\section{Conclusiones}

Se logró identificar que poco menos de la mitad presenta sobrepeso, una cuarta parte de la población presenta obesidad, en los escolares, casi la totalidad de los escolares realizan una actividad física durante el pre y post intervención.

Al final de la intervención el IMC se redujo, aumentando una tercera parte de escolares con peso saludable, y disminuyendo en un nivel significativo.

\section{Referencias}

1. MEJOR., O. M. (27 de MAYO de 2014). La estrategia de México para hacer frente a la obesidad es prometedora, dice la OCDE. Recuperado el 27 de OCTUBRE de 2016, de

http://www.oecd.org/centrodemexico/medios/laestrategiademexicoparahac erfrente alaobesidadesprometedoradicelaocde.htm

2. OMS. (2017). Obtenido de Estrategia mundial sobre régimen alimentario, actividad física y salud: http://www.who.int/dietphysicalactivity/pa/es/

3. Ortiz, Z. (Julio de 2012). UNICEF. Recuperado el 28 de octubre de 2016, de Evaluación del crecimiento de niñas y niños: http://www.unicef.org/argentina/spanish/Nutricion_24julio.pdf

4. Molina N, L. N. (3 de Diciembre de 2012). Recuperado el 27 de octubre de 2016, de Niñez intermedia: http://ninezintermedia-phd303- 
Publicación semestral Educación y Salud Boletín Científico Instituto de Ciencias de la Salud Universidad Autónoma del Estado de Hidalgo, Vol. 8, No. 15 (2019) 180-183

grupo3.blogspot.mx/2012/12/laninez-intermedia-de-6-12-anos-deedad.html

5. Flores, M. (Diciembre de 2012). Intervenciones dirigidas a disminuir/evitar el sedentarismo en los escolares. Recuperado el 4 de noviembre de 2016, de http://www.scielo.org.mx/scielo.php?pid=S166570632012000400005\&scr ipt=sci_arttext

6. Sir, A. (24 de Marzo de 2018). Organización Mundial de la Salud. Obtenido de http://apps.who.int/gb/ebwha/pdf_files/WHA69/A69_8sp.pdf

7. Laguado, J. (5 de MAYO de 2014). SCIELO. Obtenido de ESTILOS DE VIDA SALUDABLE EN ESTUDIANTES DE ENFERMERIA: http://www.scielo.org.co/pdf/hpsal/v19n1/v19n1a06.pdf 